\title{
Actitudes del profesor de Biología hacia las prácticas de enseñanza a través de TIC ${ }^{1}$ \\ Biology teachers attitudes towards the teaching practices through ICTs
}

\author{
Héctor Alexander Afanador Castañeda ${ }^{2}$
}

\section{Resumen}

Este artículo tiene como objetivo validar los ítems de escala de la entrevista de actitudes del profesor de Biología hacia las prácticas de enseñanza de la Biología a través de TIC, para ello se tuvo en cuenta un diseño metodológico interpretativo que dispuso de cuatro etapas: $1^{\circ}$ etapa de revisión documentada, $2^{\circ}$ construcción del instrumento desde la teoría, $3^{\circ}$ validación del instrumento el cual involucró validación de expertos y validación interna a partir del alfa de Cronbach y $4^{\circ}$ procesamiento donde los datos obtenidos fueron analizados a partir de criterios valorativos correspondientes a la escala Likert. El instrumento fue aplicado a 34 profesores expertos que han integrado las TIC en la enseñanza de la Biología. Los datos revelan que los ítems y las subcategorías construidas del instrumento presentan una fuerte consistencia interna que permite determinar las actitudes de los profesores y puede ser replicado en otras investigaciones.

Palabras claves: Actitudes; Profesor de Biología; TIC.

\section{Abstract}

The objective of this article is to validate the scale items of the interview on biology teacher's attitude towards teaching practices through ICTs, for which an interpretive methodological design was taken into account; it had four stages: 1 , stage of documented revision; 2, construction of the instrument from the theory, 3 , stage of instrument validation which involved validation of experts and internal validation from Cronbach's alpha, and 4 , stage of processing where the data obtained was analyzed based on the Likert scale evaluation criteria. The instrument was applied to 34 expert teachers who have integrated ICTs in biology teaching processes. The data reveals that items such as the instrument subcategories constructed present a strong internal consistency that allows to determine the teachers' attitudes which can be replicated in other research projects.

\footnotetext{
${ }^{1}$ Artículo de investigaciónLos resultados del artículo hacen parte del proyecto de investigación doctoral: Configuración de la práctica de enseñanza a través de TIC de profesores de Biología de la Secretaría de Educación Distrital, Bogotá, suscrita en el Grupo de investigación Conocimiento Profesional del Profesor de la Universidad Pedagógica Nacional. ${ }^{2}$ Mg. Didácticas de las ciencias. Miembro del Grupo de investigación Conocimiento Profesional del Profesor de Ciencias (CPPC-UPN). Docente de la Secretaría de Educación Distrital. https://orcid.org/0000-0001-9244-911X E-mail: haacster@gmail.com

* Cómo citar este artículo: Afanador, H. (2018). Actitudes del profesor de Biología hacia las prácticas de enseñanza a través de TIC. Hojas y Hablas, (16), 27-44. DOI:10.29151/ hojasyhablas.n16a2
} 


\section{Introducción}

Los Planes de Desarrollo de Colombia desde el 2002 al 2018, se han preocupado por aumentar el presupuesto de inversión económica en TIC para educación, que según Levis (2007), ponen de primeras a la adquisición de infraestructura y equipamiento, luego al entrenamiento y capacitación, que a la vez [siendo agentes externos no creados al interior de la escuela o aula] generan dinámicas administrativas diferentes al interior de la institución, asimismo dinámicas educativas diferentes entre los integrantes de la comunidad educativa (pp. 2 -3). Con respecto a la dinámica educativa, Bonilla, Murcia y Álvarez (2012) indican que la incorporación de las TIC depende de la actitud que asumen los sujetos [comunidad educativa] puesto que para unos puede ser un cambio positivo, acelerado o novedoso, mientras que para otros es lento, invasivo o inútil para las dinámicas de la escuela.

Visto desde esta óptica, la actitud del profesor influye en la integración de las TIC ya que ésta es un estado mental organizado por la experiencia donde la predisposición del sujeto ejerce una influencia directa para pensar, sentir, percibir, y comportarse ante un objeto o todas las situaciones que se relacionan con ella (Elejabarrieta \& Iñiguez 2000). En este sentido, las actitudes son variables intermedias entre nuestras ideas y la manera como las llevamos a la práctica, es decir, son el puente interactivo (de aquí hacia allá y viceversa) entre los conceptos y los procedimientos (Mosquera, 2008). De tal modo e interpretando a Gairín (1990, p. 17), la actitud no puede ser comprendida sólo en su propio término sino a través de la expresión valorativa en la que se sitúe el profesor con su acción en un contexto, es decir, el conjunto de variables de predisposición tenderá a comprender la actitud y capacidad del profesor de Biología de utilizar e integrar las TIC dentro de la práctica de enseñanza.

Algunos investigadores demuestran la importancia de profundizar en este campo como: Valdés et al. (2011), señalan que los docentes poseen actitudes positivas hacia las TIC, especialmente los de género masculino, y aquellos que cuentan con mayores posibilidades de interacción tecnológica. A pesar de que las actitudes de los maestros son positivas, son menos favorables ante los factores de factibilidad y disponibilidad por la deficiencia de los programas de capacitación, y mejor disposición de aquellos docentes que cuentan con mayores posibilidades de interacción. Asimismo, Álvarez et al. (2011) en el cual identificaron que las actitudes hacia las TIC pueden verificarse a través de la presencialidad de las TIC en el aula puesto que el proceso de adaptación y convergencia tecnológica intervienen en la dinámica del profesor frente al uso de TIC.

No obstante, al referirnos al diseño instruccional con TIC o construcción de artefactos de aprendizaje, el cual implica una mayor predisposición del sujeto hacia las TIC, también Zapata (2006), aporta datos que afirman que los docentes evidencian indiferencia ante los objetos de aprendizaje, por el temor de enfrentarse a los diseños e innovaciones tecnológicas que aportarían a sus prácticas pedagógicas; y Lustosa de Oliveira, Silva, Vega, y Galembeck (2017), demuestran que el uso de TIC contribuye a que los profesores mejoren sus actitudes hacia la aceptación de los recursos educativos.

Lo anterior confirma la necesidad de incidir en las actitudes del profesor dentro la formación tecnológica de los profesores, puesto que Bosco (2008) indica que las actitudes de los docentes son cruciales en cualquier innovación educativa, de lo contrario no podrán implementar los 
cambios curriculares de manera adecuada. Incluso cuando los maestros son competentes en una innovación [con TIC], es posible que no tengan éxito en sus aulas cuando sus actitudes no son positivas (Alazam, Bakar, Hamzah \& Asmiran, 2012).

Consecuentemente con lo anterior no puede afirmarse categorialmente, ni decir lo contrario, que todos o la mayoría de los profesores tienen actitudes positivas hacia las TIC, interpretando a Bonilla, Murcia y Álvarez (2012), el desfase entre actitud positiva y los usos reales en el desarrollo de las clases dependen de: el acceso y disponibilidad TIC que posean los sujetos, el consumismo tecnológico, incorporación tecnológica en el sujeto (profesor y estudiante) y del contexto (escuela, familia y comunidad). De tal modo, que al partir de esta premisa se establece que los profesores presentarán una diversidad de actitudes hacia las TIC que pueden agruparse en favorables o desfavorables o ampliarse a actitudes de resistencia, inclusión, aceptación e implementación como lo plantea el trabajo investigativo de Patiño y Sallé (2014) en la integración de TIC.

En el caso de la relación utilización e integración de las TIC y actitudes de los profesores de Biología hacia las TIC, la investigación de Vera, Tomka y Snežana (2014) hace referencia a las actitudes muy positivas en el uso de artefactos en la enseñanza de la Biología donde se destacan las actitudes hacia la aplicación de algún método asistido por TIC, pero agregan que la baja experiencia del profesor en la integración de las TIC incide para que se realicen prácticas de enseñanza tradicionales, complementariamente Šorgo, Verčkovnik y Kocijančič (2010) identificaron en algunos profesores de Biología que, el optar por utilizar otro recurso tecnológico o convencional para la enseñanza depende de la relación entre la actitud y el conocimiento tecnológico, que posee el profesor.

Bajo el anterior panorama de datos en actitudes de los profesores, y en especial de los profesores de Biología, es importante indagar las actitudes de estos hacia la práctica de enseñanza de la Biología a través de TIC, puesto que los recursos tecnológicos son específicos por su contenido científico [ejemplo: el microscopio digital, el laboratorio virtual, otros], el cual hace diferenciar las prácticas de enseñanza de otras disciplinas. Por lo tanto, es necesario la construcción de instrumento específico que permita obtener datos en estos profesionales ya que se considera al profesor de Biología un sujeto con un conocimiento propio especializado que requiere de los recursos tecnológicos (TIC), pero en especial, de recursos tecnológicos específicos para la enseñanza de la Biología. A continuación, el documento describe el diseño metodológico y los resultados de validación interna y externa de la entrevista de ítems con escala, así como la tendencia cualitativa de actitudes del profesor de Biología hacia las prácticas de enseñanza a través de TIC dentro de un contexto particular y de sujetos particulares.

\section{Diseño metodológico}

Esta investigación se enmarca en el paradigma interpretativo puesto que el conjunto de suposiciones (criterios valorativos) de carácter filosófico es una manera de valerse en la aproximación de la búsqueda del conocimiento y la noción que compartimos de realidad y de verdad (Páramo y Otálvaro 2006), tal sentido representa y valora un esquema de actuación y comprensión de la realidad particular (Alvira, 1982). Siendo consecuentes, la formulación del instrumento de actitudes hacia las TIC pretende la búsqueda 
de conocimiento para comprender el sentido de la acción social de la enseñanza de la Biología, es decir comprender las predisposiciones del profesor de Biología hacia las TIC desde la experiencia misma de los participantes, para este caso, las técnicas de investigación como los datos [estadísticos y tendencias] son tan solo insumos para la comprensión de la realidad simbólicamente pre-estructurada del objeto en particular [sujeto - profesor] que requiere de la función participativa del intérprete [investigador], que no da significado a lo observado, sino que hace explicita la significación dada por los participantes (Vasilachis, 2007). De tal modo se dispuso de las siguientes etapas:

Etapa de revisión documentada: se realizó una revisión de artículos que identifiquen la vigencia y relevancia de la investigación en actitudes hacia las TIC en el profesor de Biología dentro de la comunidad científica. Para ello se delimitó el periodo cronológico a 2005 - 2015.

Etapa de construcción del instrumento de actitudes hacia las TIC, la revisión documentada fue el punto de partida puesto que se tuvieron en cuenta los planteamientos de Delgado, Arrieta y Riveros (2009), los resultados de investigaciones sobre las actitudes hacia las TIC de profesores de Biología como: Boitshwarelo (2014), Šorgo, Verčkovnik y Kocijančič (2010) y Vera, Tomka y Snežana (2014); además, instrumentos de medida tales como: Oya y Sadi (2013), Schmidt, Baran, Thompson, Mishra, Koehler, y Shin (2009), para la formulación de subcategorías del instrumento y la intencionalidad de los criterios o ítems de escala, así como la obtención final de la entrevista de ítems con escala.

Etapa de validación del instrumento, este instrumento pasa por cuatro fases: $1^{\circ}$ la prueba piloto, ésta fue realizada con un instrumento de 20 ítems aplicado a 15 profesores de Biología de básica secundaria donde se tuvo en cuenta el alfa de Cronbach para la validación interna pero los resultados no satisfactorios conllevaron a los siguientes procesos; $2^{\circ}$ validación externa, en la cual se presentó el instrumento modificado ante el grupo de investigación conocimiento profesional del profesor de ciencias (CPPC) de la Universidad Pedagógica Nacional (conformado por 3 doctores, 4 doctorandos y 3 investigadores magísteres) para establecer la comprensión, legibilidad, redacción e intencionalidad; $3^{\circ}$ la validación por experto, en este proceso se presentaron al experto Edgar Orlay Valbuena Ussa los resultados de la prueba piloto, los cambios y adaptaciones incorporadas y la justificación teórica de la subcategoría con los respectivos ítems para el aval de aplicación final. $4^{\circ}$ Aplicación final a partir de una muestra de 34 profesores expertos de Biología que en su trayectoria han usado TIC en la enseñanza de la Biología.

Etapa de procesamiento, Tejedor y García (2006) y Tejedor, García y Prada (2009), recomiendan determinar la fiabilidad interna de las entrevista de ítems con escala, en ese sentido para la validación interna del instrumento se optó por el coeficiente del alfa de Cronbach con el propósito de: establecer la confiabilidad interna de la categoría, las subcategorías y los ítems que conforman la entrevista de ítems con escala de cinco respuestas, y generar homogeneización entre las subcategorías por exclusión de toda la subcategoría (se prescinde de los ítems de una subcategoría y se vuelve a calcular el valor del coeficiente del instrumento); esto permite excluir los ítems menos consistentes que afectan a las subcategorías y a la misma categoría y optimizar la restructuración de las subcategorías y obtención del instrumento final validado (Morales 2006, p.85); en 
donde se utilizó una hoja de cálculo con herramientas estadísticas.

En el análisis de comportamiento a partir de la tendencia de la escala Likert (siendo la escala totalmente en desacuerdo, en desacuerdo, indeciso, de acuerdo y totalmente de acuerdo) se incluyó los criterios valorativos de Afanador y Mosquera (2012) para un análisis dicotómico (actitudes favorables y no favorables), donde indican que la sumatoria de respuestas seleccionadas como totalmente en desacuerdo, desacuerdo e indeciso del ítem corresponda a un porcentaje mayor o igual a $45 \%$ son actitudes desfavorables. Mientras que la tendencia de la subcategoría depende del promedio porcentual de sus ítems en donde el valor puede ser igual o mayor a $65 \%$ siendo una subcategoría con predisposición positiva. Estos criterios permitirán analizar tanto los ítems individualmente como su incidencia en la subcategoría, anexo a esta escala fue importante incluir la tendencia de frecuencia por subcategorías de forma dicotómica (actitudes favorables línea roja y actitudes no favorables línea negra) para hallar las afectaciones por agrupamiento de los ítems en las subcategorías.

\section{Resultados y discusión}

La descripción de datos será en el siguiente orden: resultados bibliométricos, resultados de validación del instrumento y resultados de comportamiento de tendencia escala Likert. En cuanto a los resultados bibliométricos, se resalta que se adoptó la búsqueda de información a partir de plataformas electrónicas como: Google académico, Dialnet.com, ScieELO.org, sciencedirect.com, oei.es, ojs.pensamultimedia, eduteka. org y ries.universia.net, entre el 2005 al 2015. Los resultados asociados cuando se emplearon las palabras claves "actitudes hacia las TIC" fueron de 9130 documentos (registro de la fecha 15 de diciembre de 2015), pero al relacionar las palabras claves profesor de Biología AND y OR actitudes TIC, los resultados se redujeron drásticamente a 12 artículos científicos, de los cuales 5 artículos son de carácter particular y pertinentes al contexto escolar de básica secundaria de la enseñanza de la Biología mediada con TIC que fueron utilizados en la construcción del instrumento. En cuanto resultado de la prueba piloto del instrumento "actitudes del profesor de Biología hacia las prácticas de enseñanza de la Biología a través de TIC" a partir del coeficiente Cronbach no fue consistente o satisfactorio, puesto que su valor fue muy bajo (0.34) para los 20 ítems que conformaban la entrevista (ver tabla 1), impidiendo así la utilización del instrumento para una exploración en las actitudes de los profesores. 
Tabla 1. Coeficiente de Cronbach del instrumento "actitudes del profesor de Biología hacia las prácticas de enseñanza de Biología a través de TIC" de la prueba piloto.

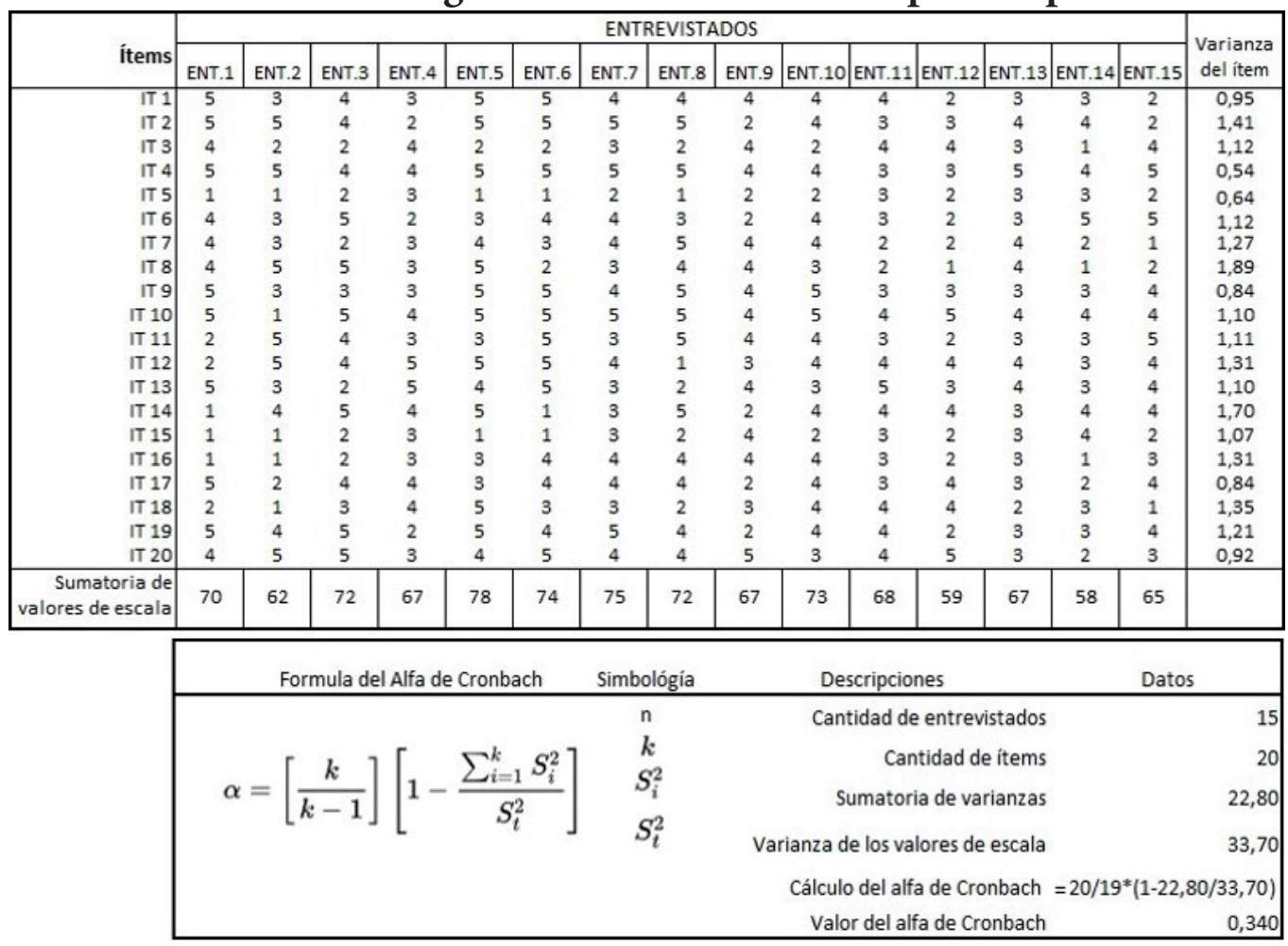

Fuente: elaboración propia.

En conformidad con los datos, se llevó a cabo la revisión de ítems del instrumento en donde se tomó la determinación de eliminar nueve ítems, ajustar los once ítems restantes (en el instrumento final son: IT1, IT5, IT6, IT7, IT9, IT11, IT23, IT10, IT24, IT32 e IT35) y aumentar la cantidad de ítems para un total de 35 . En ese sentido, el nuevo instrumento tuvo que ser llevado a

la validación externa en donde los investigadores [grupo de investigación CPPC] concordaron en ciertas incongruencias. Según los datos de la evaluación externa (ver tabla 2), se tuvieron que corregir diez ítems con anterioridad a la validación por experto con el propósito de obtener el aval para la aplicación final del instrumento.

Tabla 2. Criterios para la validación externa del instrumento "actitudes del profesor de Biología hacia las prácticas de enseñanza de Biología a través de TIC”.

\begin{tabular}{|c|l|c|c|c|}
\hline Criterios & \multicolumn{1}{|c|}{ Descripciones } & $\begin{array}{c}\text { Cantidad } \\
\text { de items } \\
\text { aceptados }\end{array}$ & $\begin{array}{c}\text { Cantidad items } \\
\text { que deben ser } \\
\text { corregidos }\end{array}$ & $\begin{array}{c}\text { Cantidad de } \\
\text { items no } \\
\text { aceptados }\end{array}$ \\
\hline Comprensión & $\begin{array}{l}\text { La sentencia o afirmación presenta sentido o significado } \\
\text { donde el entrevistado involucra o relaciona su saber y del } \\
\text { interés propio sobre las TIC. }\end{array}$ & 29 & $\begin{array}{c}4 \\
\text { Ítems: } 2 ; 13 ; \\
30 ; 34 .\end{array}$ & 0 \\
\hline Redacción & $\begin{array}{l}\text { Las sentencias presentan consistencia y cohesión textual } \\
\text { en donde se evidencia que lo que se quiere escribir esta } \\
\text { debidamente ordenado y no genera doble sentido. }\end{array}$ & 30 & $\begin{array}{c}4 \\
\text { Ítems: } 3 ; 19 ; \\
22 ; 28 .\end{array}$ & 0 \\
\hline Intencionalidad & $\begin{array}{l}\text { En cada una de las sentencias evidencia o se explicita el } \\
\text { objetivo o propósito que se persigue, permitiendo al } \\
\text { entrevistado estar de acuerdo o en desacuerdo. }\end{array}$ & 32 & $\begin{array}{c}3 \\
\text { Ítems: } 15 ; 22 ; \\
34 .\end{array}$ & 0 \\
\hline Legibilidad & $\begin{array}{l}\text { Cada una de las sentencias, como el mismo instrumento, } \\
\text { muestran que son insumos sin errores en letra } \\
\text { presentación y espacialidad que puede ser utilizado en } \\
\text { formato impreso o digital, además permite una lectura } \\
\text { fácil y rápida. }\end{array}$ & 34 & $\begin{array}{c}1 \\
\text { Ítems: } 19\end{array}$ & 0 \\
\hline
\end{tabular}

Fuente: elaboración propia. 
Los resultados de la aplicación final del instrumento se describen en el siguiente orden: validación interna del instrumento adaptado de actitudes y descripciones de comportamiento de tendencia de las actitudes.

En la aplicación final del instrumento (35 ítems), los datos obtenidos de confiabilidad interna establecieron que el aumento en cantidad y perfeccionamiento de los ítems contribuyó al aumento del valor del coeficiente interno (0.702), pero este valor por sí solo no es factor determinante para la confiablidad interna de cada una de las subcategorías. Entonces, al calcular el coeficiente interno de cada subcategoría se identificó que la subcategoría 2 y la subcategoría 3 tienen valores muy bajos (insatisfactorios) para su replicación (ver tabla 3).

Tabla 3. Coeficiente del alfa de Cronbach en la categoría y subcategorías de las actitudes del profesor de Biología hacia las prácticas de enseñanza de la Biología a través de TIC.

\begin{tabular}{ccccc}
$\mathbf{n}^{\circ}$ ítems & Categoría & $\begin{array}{c}\text { Subcategoría } \\
\text { uno }\end{array}$ & $\begin{array}{c}\text { Subcategoría } \\
\text { dos }\end{array}$ & $\begin{array}{c}\text { Subcategoría } \\
\text { tres }\end{array}$ \\
\hline 35 & 0.702 & 0.652 & 0.432 & 0.387
\end{tabular}

Fuente: elaboración propia.

Para aumentar la validación interna del instrumento se excluyeron los ítems que presentaron valores altos en el coeficiente de Cronbach cuando se prescinde del mismo. En ese sentido se excluyeron cinco ítems, y fueron: ítem 28 (prefiero elegir la práctica de enseñanza de la Biología sin TIC que una práctica de enseñanza de Biología con TIC), ítem 3 (los recursos didácticos son requisitos para desarrollar las prácticas de enseñanza de Biología), ítem 22 (es preferible hacer la prác- tica de enseñanza de la Biología en el aula normal sin TIC, que hacer la práctica de enseñanza de la Biología en el aula de informática), ítem 27 (llevar los equipos tecnológicos al salón de clase entorpece u obstaculiza la práctica de enseñanza de la Biología), e ítem 15 (los profesores no requieren conocimientos sobre las TIC para realizar innovaciones en las prácticas de enseñanza de la Biología), ver tabla 4.

\section{Tabla 4. Valores del alfa de Cronbach excluyendo el ítem para todo el instrumento}

\begin{tabular}{cccccc}
\multicolumn{2}{c}{ Categoría uno } & \multicolumn{2}{c}{ Categoría dos } & \multicolumn{2}{c}{ Categoría tres } \\
\hline İtems & Alfa de Cronbach & İtems & Alfa de Cronbach & Ítems & Alfa de Cronbach \\
\hline 1 & 0.660 & 2 & 0.696 & 23 & 0.708 \\
\hline 5 & 0.685 & 3 & 0.707 excluido & 32 & 0.687 \\
\hline 6 & 0.715 & 18 & 0.694 & 7 & 0.709 \\
\hline 16 & 0.690 & 20 & 0.683 & 8 & 0.685 \\
\hline 28 & 0.736 excluido & 24 & 0.679 & 10 & 0.682 \\
\hline 4 & 0.724 & 26 & 0.702 & 12 & 0.690 \\
\hline 9 & 0.669 & 29 & 0.701 & 15 & 0.730 excluido \\
\hline 11 & 0.665 & 14 & 0.688 & 21 & 0.696 \\
\hline 13 & 0.678 & 30 & 0.707 & 25 & 0.689 \\
\hline 17 & 0.651 & 22 & 0.739 excluido & 34 & 0.704 \\
\hline 19 & 0.662 & 27 & 0.730 excluido & 35 & 0.685 \\
\hline \multicolumn{7}{r}{} & 31 & 0.687 & & \\
\hline
\end{tabular}

Fuente: elaboración propia. 
Sin embargo, el aumento de la confiabilidad interna del instrumento no indica que las subcategorías construidas y la relación entre ellas mismas sea homogénea en su consistencia y fiabilidad interna. Nótese en la tabla 5, que aumentó la validación interna (fiabilidad interna y replicación) del instrumento (categoría) y en cada una de las subcategorías, pero los valores internos del alfa de Cronbach de la subcategoría dos y subcategoría tres $(<0.55)$ y el valor de homogeneización de una de las subcategorías (exclusión de la subcategoría uno: < 0.5); no fue lo suficientemente aceptable o satisfactorio.

Tabla 5. Valores del coeficiente del alfa de Cronbach en la categoría y las subcategorías a partir de la exclusión de ítems y exclusión de subcategorías.

\begin{tabular}{ccccc}
$\mathbf{n}^{\circ}$ ítems & Categoría & $\begin{array}{c}\text { Subcategoría } \\
\text { uno }\end{array}$ & $\begin{array}{c}\text { Subcategoría } \\
\text { dos }\end{array}$ & $\begin{array}{c}\text { Subcategoría } \\
\text { tres }\end{array}$ \\
\hline 30 & 0.79 & 0.767 & 0.545 & 0.438 \\
\hline Exclusión de la subcategoría & 0.471 & 0.758 & 0.768
\end{tabular}

Fuente: elaboración propia.

Los anteriores resultados determinaron la necesidad de seleccionar y excluir nuevos ítems desde las mismas subcategorías para favorecer los coeficientes internos de las subcategorías y los valores de homogeneización entre subcategorías. Entonces, se decidió aplicar de nuevo el alfa de Cronbach excluyendo ítems por subcategoría (ver tabla 6).

Tabla 6. Valores del alfa de Cronbach excluyendo el ítem por cada subcategoría

Categoría uno

Categoría dos

Categoría tres

\begin{tabular}{cccccc}
\hline Ítems & Alfa de Cronbach & Ítems & Alfa de Cronbach & Ítems & Alfa de Cronbach \\
\hline 1 & 0.711 & 2 & 0.565 & 23 & 0.318 \\
\hline 5 & 0.728 & 18 & 0.552 & 32 & 0.554 excluido \\
\hline 6 & 0.835 excluido & 20 & 0.432 & 7 & 0.513 \\
\hline 16 & 0.737 & 24 & 0.570 & 8 & 0.335 \\
\hline 4 & 0.807 & 26 & 0.505 & 10 & 0.468 excluido \\
\hline 9 & 0.718 & 29 & 0.466 & 12 & 0.325 \\
\hline 11 & 0.720 & 14 & 0.557 excluido & 21 & 0.504 \\
\hline 13 & 0.727 & 30 & 0.584 excluido & 25 & 0.299 \\
\hline 17 & 0.695 & 31 & 0.467 & 34 & 0.361 \\
\hline 19 & 0.740 & 33 & 0.455 & 35 & 0.299
\end{tabular}

Fuente: elaboración propia. 
Los anteriores ítem excluidos [intencionadamente] permitieron el aumento significativo de los coeficientes del alfa de Cronbach en cada una de las subcategorías, así mismo, los valores de homogeneización del coeficiente del alfa de Cronbach entre las subcategorías del instrumento y el coeficiente interno del instrumento (ver tabla 7).

Tabla 7. Valores ajustados del coeficiente del alfa de Cronbach en la categoría y las subcategorías a partir de la exclusión de ítems y exclusión de subcategorías

\begin{tabular}{ccccc} 
nítems & Categoría & $\begin{array}{c}\text { Subcategoría } \\
\text { uno }\end{array}$ & $\begin{array}{c}\text { Subcategoría } \\
\text { dos }\end{array}$ & $\begin{array}{c}\text { Subcategoría } \\
\text { tres }\end{array}$ \\
\hline 25 & 0.842 & 0.835 & 0.601 & 0.619 \\
\hline Exclusión de la subcategoría & 0.731 & 0.776 & 0.784
\end{tabular}

Fuente: elaboración propia.

Según la tabla 7, el instrumento como categoría adquirió un coeficiente de alfa de Cronbach satisfactorio (>0.8) luego de prescindir de 10 ítems. Esto además benefició los coeficientes internos de las subcategorías dos y tres $(>0.6)$, siendo estos aceptables y en la subcategoría uno satisfactorio $(>0.8)$. De igual manera, valores de homogeneización en las subcategorías fueron valores satisfactorios $(>0.7)$ cuando se prescinde de los ítems de la subcategoría. Estos resultados permitieron que el instrumento final (categoría) conste de 25 ítems distribuidos en tres subcategorías (subcategoría uno tiene nueve ítems, la subcategoría dos tiene ocho ítems y la subcategoría tres tiene ocho ítems). A continuación, se justifica cada una de las subcategorías del instrumento:

La subcategoría uno o actitudes "autoconcepto en el uso de TIC para la práctica de enseñanza de la Biología”, se justifica a partir de los planteamientos de Fernández, Álvarez, Velásquez y Ruiz (2002) y Martínez (2010), en el cual concuerdan que, el concepto de sí mismo refleja la forma en que el sujeto se relaciona con el objeto, de tal modo, que se genera una predisposición. En ese sentido los ítems que constituyen esta subcategoría son (ver tabla 8): 
Tabla 8. Ítems de la subcategoría actitudes del profesor sobre autoconcepto en el uso de TIC para la práctica de enseñanza de la Biología.

\section{Ítems}

1. Soy un docente entusiasta que busca en cada momento integrar las TIC a las prácticas de enseñanza de la Biología.

5. Me preocupo por tener dominio suficiente sobre los artefactos tecnológicos de la institución que me permitan enseñarles a otros cómo se emplean.

16. Analizo toda la información que se encuentra en los recursos digitales antes de llevarla a la práctica de enseñanza de la Biología

4. Recurro más al uso de las TIC que al uso del experimento cuando enseño contenidos (abstractos y complejos) en las prácticas de enseñanza de Biología.

9. Recurro más al uso de las TIC que al uso del tablero cuando enseño contenidos (abstractos y complejos) en la práctica de enseñanza de la Biología.

11. Recurro más al uso de las TIC que al uso del texto impreso cuando enseño contenidos (abstractos y complejos) en la práctica de enseñanza de la Biología.

13. Por lo general uso las TIC en la práctica de enseñanza de Biología para establecer procesos comunicativos o transmisión de información.

17. Recurro más al uso de evaluaciones en línea que al uso de evaluaciones impresas en la práctica de enseñanza de Biología.

19. Recurro más a la información en la web que a la información de los libros impresos cuando enseño contenidos (abstractos y complejos) en la práctica de enseñanza de la Biología.

Fuente: elaboración propia.

Es relevante indicar que, para las actitudes hacia la integración de las TIC en las prácticas de enseñanza de la Biología, desde los postulados de Escorcia y James, (2014); UNESCO, (2013 p. 34) y Yarto, (2010), pretende afirmar la predisposición del sujeto por encontrarle a las TIC un sentido, y que su actitud frente al uso de las TIC obedece a las necesidades de cada sujeto para la enseñanza de la Biología dentro de un contexto particular. En ese sentido son los siguientes ítems de la subcategoría (ver tabla 9):

Tabla 9. Ítems de la subcategoría actitudes del profesor hacia la integración de las TIC en la práctica de enseñanza de la Biología. 


\section{Ítems}

26. Un buen recurso tecnológico disminuye la cantidad de explicaciones o discursos del profesor en la práctica de enseñanza de Biología mediadas con TIC.

29. La integración de las TIC en la práctica de enseñanza de la Biología genera mayor apertura cognitiva hacia el conocimiento científico.

33. El buen uso y la excelente integración de las TIC en la práctica de enseñanza de la Biología contribuyen a que el profesor no repita explicaciones.

2. Es muy relevante el uso de las TIC para las prácticas de enseñanza de la Biología.

20. En la práctica de enseñanza de la Biología mediadas con TIC es importante reconocer que las

TIC son agentes innovadores.

18. La práctica de enseñanza de la Biología tiene mayor éxito cuando se realiza con TIC.

24. Las prácticas de enseñanza de la Biología son exitosas cuando se realiza mayor cantidad de experimentos virtuales que con experimentos presenciales.

31. Actualmente, son más aceptadas las prácticas de enseñanza de la Biología que innoven con TIC.

Fuente: elaboración propia.

Para la subcategoría actitudes del profesor de Biología hacia conocimiento de TIC para las prácticas de enseñanza de la Biología", precisa que las acciones que se realizan sobre las TIC son indicadores de conocimiento ya que éstas son utilizables de muchas maneras diferentes (Koehler \& Mishra, 2009; Cavas Cavas, Karaoglan, \& Kisla 2009), por lo tanto el profesor de Biología presenta predisposición frente aspectos de formación, construcción, organización, dominio y comprensión; de las TIC como artefactos de mediación de la práctica de enseñanza. Los ítems que conforman esta categoría son (ver tabla 10):

\section{Tabla 10. Ítems de la subcategoría actitudes del profesor hacia el conocimiento de TIC para práctica de enseñanza de la Biología. \\ Ítems}

23. Es importante en las prácticas de enseñanza de la Biología buscar recursos tecnológicos para explicar fenómenos naturales.

35. En la práctica de enseñanza de la Biología es importante que el profesor tenga dominio tecnológico

(TIC) para que pueda desarrollar correctamente el contenido didáctico.

12. Es importante para el profesor de Biología poseer conocimientos instrumentales sobre las TIC.

21. El conocimiento instrumental sobre las TIC es imprescindible cuando se construye una secuencia de actividades para la práctica de enseñanza de la Biología.

8. La actualización o formación en TIC genera innovación en las prácticas de enseñanza de la Biología

25. Cuando se es profesor de Biología debe tener disposición para la formación en TIC.

7. Es importante observar directamente a otro profesor de Biología para saber cómo integrar las TIC en las prácticas de enseñanza de la Biología.

34. Los conocimientos sobre los recursos tecnológicos que tienen los profesores de Biología son suficientes para diseñar prácticas de enseñanza de Biología mediadas con TIC. 
Se discute que los resultados obtenidos del coeficiente del alfa de Cronbach en las primeras validaciones internas del instrumento no permitieron el uso del instrumento porque las subcategorías construidas no fueron lo suficientemente consistente (prueba piloto 0.34 y aplicación final en subcategoría dos 0.43 y en subcategoría tres 0.38; del instrumento sin ajustar). Según la escala de Huh, Delorme y Reid, (2006) y Nunnally, (1967), son valores inaceptables cuando se pretende realizar una investigación de carácter exploratoria.

En concordancia con lo anterior, la aplicación final de la entrevista de ítems con escala requerirá de acciones como: la modificación, la adaptación, la reformulación del instrumento, y en especial la exclusión de ítems; para mejorar la validación interna. Tal sentido de rediseño, permitió que el instrumento de actitudes del profesor de Biología hacia las prácticas de enseñanza con TIC optimizará sus valores a partir del coeficiente del alfa de Cronbach en donde se alcanzó un valor satisfactorio $(>0.8)$ para la categoría y valores aceptables $(>0.6)$ en las subcategorías, los cuales se encuentran dentro de la escala de valor del coeficiente recomendado por Nunnally (1967), Nunnally y Bernstein (1978) y Huh, Delorme y Reid (2006) para primeras etapas de investigación e investigaciones de análisis exploratorio estándar. Además, los resultados del alfa de Cronbach para la homogeneización del instrumento [valores de exclusión de las subcategorías > 0.7] demostraron que cada una de las subcategorías puede ser analizada de forma dependiente (análisis globales) e independiente (análisis particulares) para determinar las actitudes del profesor de Biología hacia las prácticas de enseñanza a través de TIC.

En cuanto a los resultados del comportamiento de la tendencia escala Likert en las actitudes del profesor de Biología hacia las prácticas de enseñanza de la Biología a través de TIC a partir de los criterios valorativos indican diferentes tendencias en los ítems y diferencias en promedios de tendencia entre las subcategorías uno y dos, con respecto a la subcategoría tres; permitiendo generar subdivisiones para análisis más precisos dentro de categoría. A continuación, se describe la tendencia de cada una de las subcategorías.

Los datos en la subcategoría "actitudes del profesor sobre autoconcepto en el uso de TIC para la práctica de enseñanza de la Biología”, según criterios valorativos se identificó que los ítems IT1, IT5, IT16, IT19 e IT13 responden actitudes favorables o positivas mientras que los ítems IT4, IT9, IT11 e IT17 pertenecen a actitudes desfavorables o negativas (ver gráfica 1), ya que estos últimos están por encima del 45\%. Sin embargo, el agrupamiento de los tres primeros ítems (IT1, IT5, IT16) determinan que los profesores de Biología presentan una predisposición positiva hacia las TIC a partir del entusiasmo, el dominio y la preparación [grupo de actitudes de autoconcepto del interés hacia las TIC], muy diferente ocurre con las actitudes negativas del agrupamiento de actitudes de autoconcepto hacia el uso de las TIC en el aula (IT4, IT9, IT1, IT17).

Gráfica 1. Tendencia de actitudes del profesor sobre autoconcepto en el uso de TIC para las prácticas de enseñanza de la Biología.

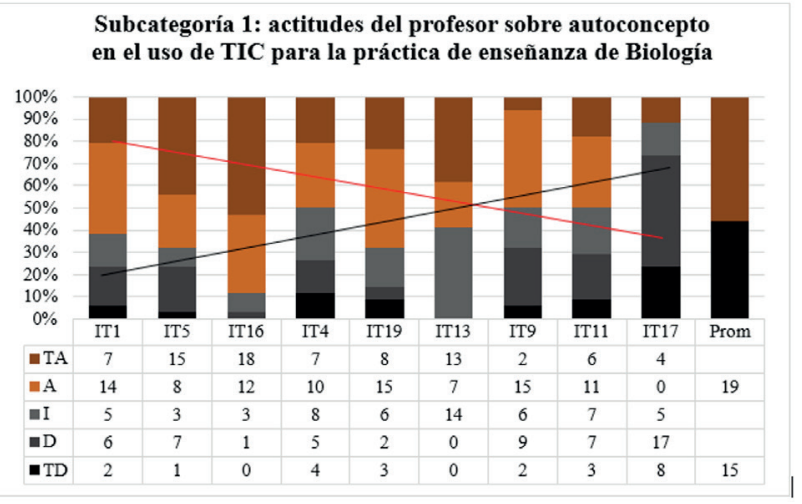


En cuanto al entrecruzamiento de las líneas de tendencia de actitudes positivas (línea roja) y actitudes negativas (línea negra) demuestran que la tendencia de la subcategoría 1 no es lo suficientemente positiva o favorable, gracias a que los profesores aún tienden a utilizar libros impresos, evaluaciones manuales o impresas, trabajo de laboratorio presencial, como también lo indica el promedio de tendencia en actitudes favorables 56 \% (19 profesores) estando por debajo del criterio valorativo para subcategorías. Entonces se establece para la subcategoría 1 , fue afectada por los ítems actitudinales relacionados con autoconcepto del uso de recursos tecnológicos y no por las actitudes de autoconcepto de interés por las TIC.

Para el caso de la subcategoría "actitudes del profesor hacia la integración de las TIC en la práctica de enseñanza de la Biología”, las actitudes de los ítems IT26, IT29, IT33, IT2, IT20 e IT18 presentaron tendencia favorable, mientras que las actitudes de los ítems IT24 e IT31 fueron tendencias no favorables o negativas (ver gráfica 2). Los datos de la subcategoría 2 indican claramente que el agrupamiento de las actitudes de funcionalidad de las TIC (IT26, IT29, IT33) son favorables en la mayoría de los profesores de Biología porque contribuye con explicaciones del contenido, la apertura cognitiva y al no desgastarse con explicaciones, mientras que el agrupamiento de las actitudes de la importancia de la implementación de las TIC existen tendencias de actitudes positivas (IT2, IT20, IT18) que resaltan la relevancia, el éxito y la innovación de éstas, no fueron los suficientemente fuertes para contrarrestar las actitudes negativas (IT24, IT31) ya que los profesores mantienen su predisposición hacia la implementación de actividades de enseñanza que vienen trabajando durante muchos años [tradicionales] y que no abandonan, como lo es el trabajo de laboratorio presencial y proyectos de aula que no incluyen las

\section{Gráfica 2. Tendencia de actitudes del profesor hacia la integración de las TIC en la práctica de enseñanza de la Biología.}

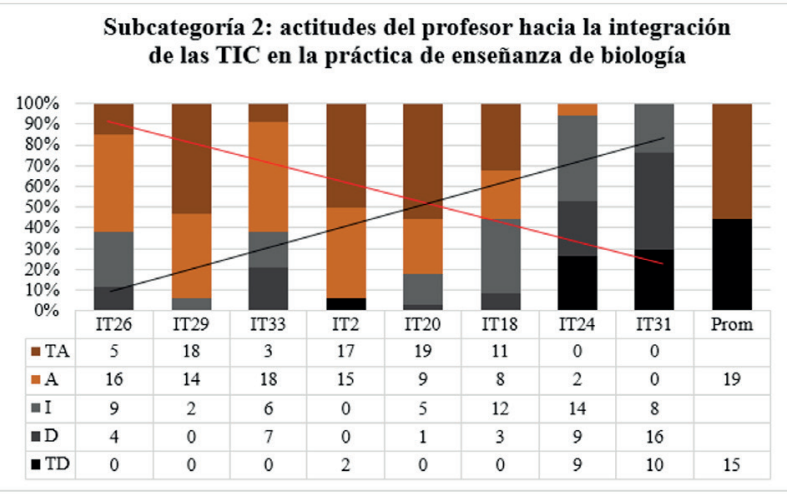

Además, el entrecruzamiento de las líneas de tendencia denota que las actitudes de los ítems IT24 e IT31 afectan directamente el agrupamiento de actitudes importancia de la implementación de TIC, pero a la vez afectan poderosamente la subcategoría, es así como el promedio de tendencia favorable de esta subcategoría tan solo fue del $57 \%$. En ese sentido la categoría 2, "actitudes hacia la integración de las TIC en las prácticas de enseñanza de la Biología", fue afectada por el agrupamiento de ítems relacionadas con las actitudes de implementación de las TIC, más no por las actitudes de funcionalidad de las TIC.

En la subcategoría 3, se establece que existe una tendencia favorable en la mayoría de las actitudes de los profesores de Biología (IT12, IT21, IT23, IT35, IT8, IT25 e IT34), mientras que solo la actitud IT17 presentó tendencia negativa (ver gráfica 3). Los datos de la subcategoría 3, indican en el agrupamiento de actitudes relacionadas con el componente tecnológico (IT23, IT35, IT12, IT21) que los profesores de Biología en su mayoría tienen predisposición positiva por el conocimiento sobre recursos tecnológicos y conocimiento instrumental de TIC además de su relación con lo didáctico y la secuencia de actividades, de igual manera, se expresa una tendencia positiva en las actitudes del agrupamiento formación en TIC en los mismos profesores. 
Gráfica 3.Tendencia de subcategoría actitudes del profesor hacia el conocimiento de TIC para la práctica de enseñanza de la Biología.

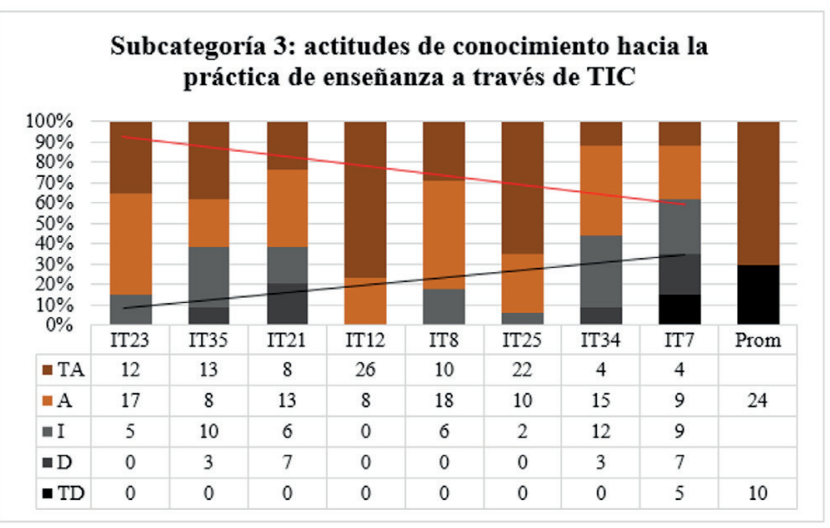

En las líneas de tendencia de actitudes, se identificó que éstas no se entrecruzan permitiendo afirmar que toda la subcategoría tiende a una predisposición positiva como lo corrobora el porcentaje del 72\% (24 profesores). Sin embargo, es importante indicar en la subcategoría "actitudes del profesor hacia el conocimiento de TIC para las prácticas de enseñanza de la Biología", que las actitudes relacionadas de construcción colectiva a partir de TIC (IT7) pueden afectar negativamente las actitudes relacionadas con formación del profesor de Biología (IT34).

En la tendencia de los ítems de escala desde la perspectiva de los criterios valorativos de la escala Likert es importante destacar que los porcentajes, tanto de los ítems como de las mismas subcategorías, distan muchos de los resultados muy positivos que indican Levent (2010), Nechita y Timofti (2011) y Vera, Tomka y Snežana (2014), cuando se refiere al uso de artefactos e integración de una modalidad asistida por TIC, puesto que el instrumento que se construyó para su validación permite identificar relaciones entre subcategorías y entre los mismos ítems de escala de la misma subcategoría profundizando en las actitudes del profesor de Biología hacia las prácticas de enseñanza de la Biología a través de TIC ya que hay que diferenciar el uso rutinario o cotidiano de las TIC de la enseñanza de la Biología con TIC.
Esta diferenciación puede compartir cierta similitud con el planteamiento de Boitshwarelo (2014), Cavas Cavas, Karaoglan y Kisla (2009), y Paredes y Dias (2012), pues consideran que existe una relación muy estrecha entre las actitudes hacia las TIC y la secuencia de utilización de las TIC como requisito para que las TIC se integren en la construcción de conocimiento con el otro [par académico]. Según la tendencia puede plantearse como hipótesis para la categoría "actitudes del profesor de Biología hacia las prácticas de enseñanza de la Biología a través de TIC"; que los profesores de biología tienen mayores predisposiciones positivas en la integración de las TIC (subcategoría 2) desde lo funcional puesto que su conocimiento de las TIC (subcategoría 3) es de carácter instrumental, debido a que sus actitudes de autoconcepto sobre las TIC (subcategoría 1) son de orden motivacional. Mientras que las actitudes no favorables del profesor de Biología responden a que su autoconcepto de las TIC en el uso de artefactos tecnológicos afecta la relevancia que tiene la integración de las TIC en las prácticas de enseñanza (subcategoría 2) debido a que su conocimiento no existe una suficientemente formación del profesor de Biología, específicamente en la construcción colectiva de prácticas de enseñanza de la Biología a través de TIC (subcategoría 3).

\section{Conclusiones}

Se resalta la importancia de la realización del diseño metodológico, así como la utilización del alfa de Cronbach en la validación interna del instrumento de actitudes del profesor de Biología hacia las prácticas de enseñanza de la Biología a través de TIC, porque permitió el perfeccionamiento de la categoría actitudes y sus respectivas subcategorías, además la fiabilidad y selección por exclusión del ítem influye en la pertinencia de las subcategorías y asegura que el instrumento sea fiable para la aplicación.

El utilizar criterios valorativos en el instrumento de actitudes posibilita un análisis interpre- 
tativo más profundo que permite identificar aspectos relevantes de predisposición en autoconcepto (no tan favorables), predisposición en integración (no tan favorables) y predisposición en conocimiento de las TIC (favorables) en profesores de Biología [así sea en una muestra de 34 profesores]. Cabe resaltar que al interior del instrumento existe una multiplicidad de respuestas que deben ser investigadas desde un planteamiento de estudio de caso, pues cada profesor presenta una variedad de actitudes por cada una de las divisiones de la subcategoría. Para este propósito se presenta el instrumento ajustado y validado gracias a este proceso (ver tabla 11).

\section{Tabla 11. Entrevista de ítems con escala de "actitudes del profesor de Biología hacia las prácticas de enseñanza a través de TIC"}

\begin{tabular}{|c|c|c|c|c|c|}
\hline ÍIEMS & $\begin{array}{c}\text { Totalmente } \\
\text { en } \\
\text { desacuerdo }\end{array}$ & $\begin{array}{c}\text { En } \\
\text { desacuerdo }\end{array}$ & Indeciso & $\begin{array}{c}\mathrm{De} \\
\text { acuerdo }\end{array}$ & $\begin{array}{l}\text { Totalmente } \\
\text { de acuerdo }\end{array}$ \\
\hline \multicolumn{6}{|l|}{$\begin{array}{l}\text { Soy un docente entusiasta que busca en cada momento integrar las TIC a } \\
\text { las prácticas de enseñanza de la Biologia. }\end{array}$} \\
\hline \multicolumn{6}{|l|}{$\begin{array}{l}\text { Es muy relevante el uso de las TIC para las prácticas de enseñanza de la } \\
\text { Biologia. }\end{array}$} \\
\hline \multicolumn{6}{|l|}{$\begin{array}{l}\text { Recurro más al uso de las TIC que al uso del experimento cuando enseño } \\
\text { contenidos (abstractos y complejos) en las prácticas de enseñanza de } \\
\text { Biologia. }\end{array}$} \\
\hline \multicolumn{6}{|l|}{$\begin{array}{l}\text { Me preocupo por tener dominio suficiente sobre los artefactos tecnológicos } \\
\text { de la institución que me permitan enseñarles a otros cómo se emplean. }\end{array}$} \\
\hline \multicolumn{6}{|l|}{$\begin{array}{l}\text { Es importante observar directamente a otro profesor de Biologia para saber } \\
\text { cómo integrar las TIC en las prácticas de enseñanza de la Biologia. }\end{array}$} \\
\hline \multicolumn{6}{|l|}{$\begin{array}{l}\text { La actualización o formación en TIC genera innovación en las prácticas de } \\
\text { enseñanza de la Biologia. }\end{array}$} \\
\hline \multicolumn{6}{|l|}{$\begin{array}{l}\text { Recurro más al uso de las TIC que al uso del tablero cuando enseño } \\
\text { contenidos (abstractos y complejos) en la práctica de enseñanza de la } \\
\text { Biologia. }\end{array}$} \\
\hline \multicolumn{6}{|l|}{$\begin{array}{l}\text { Recurro más al uso de las TIC que al uso del texto impreso cuando enseño } \\
\text { contenidos (abstractos y complejos) en la práctica de enseñanza de la } \\
\text { Biologia. }\end{array}$} \\
\hline \multicolumn{6}{|l|}{$\begin{array}{l}\text { Es importante para el profesor de Biologia poseer conocimientos } \\
\text { instrumentales sobre las TIC. }\end{array}$} \\
\hline \multicolumn{6}{|l|}{$\begin{array}{l}\text { Por lo general uso las TIC en la práctica de enseñanza de Biologia para } \\
\text { establecer procesos comunicativos o transmisión de información. }\end{array}$} \\
\hline \multicolumn{6}{|l|}{$\begin{array}{l}\text { Analizo toda la información que se encuentra en los recursos digitales antes } \\
\text { de llevarla a la práctica de enseñanza de la Biologia. }\end{array}$} \\
\hline \multicolumn{6}{|l|}{$\begin{array}{l}\text { Recurro más al uso de evaluaciones en linea que al uso de evaluaciones } \\
\text { impresas en la práctica de enseñanza de Biologia. }\end{array}$} \\
\hline \multicolumn{6}{|l|}{$\begin{array}{l}\text { La práctica de enseñanza de la Biologia tiene mayor éxito cuando se realiza } \\
\text { con TIC. }\end{array}$} \\
\hline \multicolumn{6}{|l|}{$\begin{array}{l}\text { Recurro más a la información en la web que a la información de los libros } \\
\text { impresos cuando enseño contenidos (abstractos y complejos) en la práctica } \\
\text { de enseñanza de la Biologia. }\end{array}$} \\
\hline \multicolumn{6}{|l|}{$\begin{array}{l}\text { En la práctica de enseñanza de la Biologia mediadas con TIC es importante } \\
\text { reconocer que las TIC son agentes innovadores. }\end{array}$} \\
\hline \multicolumn{6}{|l|}{$\begin{array}{l}\text { El conocimiento instrumental sobre las TIC es imprescindible cuando se } \\
\text { construye una secuencia de actividades para la práctica de enseñanza de la } \\
\text { Biologia. }\end{array}$} \\
\hline \multicolumn{6}{|l|}{$\begin{array}{l}\text { Es importante en las prácticas de enseñanza de la Biologia buscar recursos } \\
\text { tecnológicos para explicar fenómenos naturales. }\end{array}$} \\
\hline \multicolumn{6}{|l|}{$\begin{array}{l}\text { Las prácticas de enseñanza de la Biologia son exitosas cuando se realiza } \\
\text { mayor cantidad de experimentos virtuales que con experimentos } \\
\text { presenciales. }\end{array}$} \\
\hline \multicolumn{6}{|l|}{ Cuando se es profesor de Biologia debe tener disposición para la formación } \\
\hline \multicolumn{6}{|l|}{$\begin{array}{l}\text { Un buen recurso tecnológico disminuye la cantidad de explicaciones o } \\
\text { discursos del profesor en la práctica de enseñanza de Biologia mediadas con } \\
\text { TIC. }\end{array}$} \\
\hline \multicolumn{6}{|l|}{$\begin{array}{l}\text { La integración de las TIC en la práctica de enseñanza de la Biologia genera } \\
\text { mayor apertura cognitiva hacia el conocimiento cientifico. }\end{array}$} \\
\hline \multicolumn{6}{|l|}{$\begin{array}{l}\text { Actualmente, son más aceptadas las prácticas de enseñanza de la Biologia } \\
\text { que innoven con TIC. }\end{array}$} \\
\hline \multicolumn{6}{|l|}{$\begin{array}{l}\text { El buen uso y la excelente integración de las TIC en la práctica de enseñanza } \\
\text { de la Biologia contribuyen a que el profesor no repita explicaciones. }\end{array}$} \\
\hline $\begin{array}{l}\text { Los conocimientos sobre los recursos tecnológicos que tienen los } \\
\text { profesores de Biologia son suficientes para diseñar prácticas de enseñanza } \\
\text { de Biologia mediadas con TIC. }\end{array}$ & & & & & \\
\hline
\end{tabular}

Fuente: elaboración propia. 
Dentro de las recomendaciones a seguir [también recomendación para otros investigadores] es la construcción de entrevistas semi-estructuradas para profundizar en las subcategorías y para obtener datos asociados a otras categorías (políticas educativas en TIC, integración de las TIC en el aula, conocimiento TIC, pedagógico y didáctico) en el profesor de Biología; o estudios correlacionales, pues no se puede universalizar si los profesores tienen actitudes favorables, ni mucho menos, desfavorables hacia las TIC, puesto que factores extrínsecos e intrínsecos pueden afectar las actitudes del profesor de Biología hacia las prácticas de enseñanza de la Biología a través de TIC ya que éstas no son estables.

\section{Referencias}

Afanador, H. \& Mosquera, C. (2012). Valoración de actitudes hacia la ciencia y actitudes hacia el aprendizaje de la Biología en educación secundaria. Revista Bio-grafía. 5 (8), 32 - 49.

Alazam, A, Bakar, A. Hamzah, R \& Asmiran, S. (2012). Teachers' ICT Skills and ICT Integration in the Classroom: The Case of Vocational and Technical Teachers in Malaysia. Scientific Research. 3, 70-76.

Álvarez, S. Cuéllar, C. López, B. Adrada, C. Anguiano, R. Bueno, A. Comas, I. \& Gómez, S. (2011). Actitudes de los profesores ante la integración de las TIC en la práctica docente. Estudio de un grupo de la Universidad de Valladolid. Revista electrónica de Tecnología Educativa. 35, 1-19. Recuperado de http://www.edutec.es/revista/index.php/ edutec-e/article/view/416/152.

Alvira, F. (1983). Perspectiva cualitativa y perspectiva cuantitativa en la metodología sociológica. Revista Española de Investigaciones Sociológicas. 22, 53-75.

Bonilla, O. Murcia, E. \& Álvarez, J. (2012). Estado del arte de la educación virtual en Risaralda: Preliminares conceptuales que orientan el proceso investigativo. Revista Textos \& Sentidos, 6, 163-187.
Boitshwarelo, B. (2009). Exploring Blended Learning for Science Teacher Professional Development in an African Context. The International Review of Research in Open and Distributed Learning. 10, (4). Recuperado de http://www.irrodl.org/index.php/irrodl/ article/view/687/1321

Bosco, A. (2008). Las tecnologías de la información y la comunicación en la formación del profesorado: lineamientos, actualidad y prospectiva. Razón y Palabra, 13 (63). Recuperado de http://www.redalyc.org/articulo. oa? id=199520798002

Cavas, B. Cavas, P. Karaoglan, B. \& Kisla T. (2009). A study on science teachers' attitudes toward information and communication technologies in education. The Turkish Online Journal of Educational Technology, 8 (2), 1-13.

Delgado, M. Arrieta, X. \& Riveros, V. (2009). Uso de las TIC en educación, una propuesta para su optimización. Revista Omnia. 15 (3), 5877.

Elejabarrieta, F.J \& Iñiguez, L. (2000). Construcción de escalas de actitud tipo Thurst y Likert. Revista electrónica la Sociología en sus escenarios. 4, 1-50. Recuperado de http:// aprendeenlinea.udea.edu.co/revistas/index. $\mathrm{php} / \mathrm{ceo} /$ article/view/6564/6015

Escorcia, L. \& Jaimes, C. (2015). Tendencias de uso de las TIC en el contexto escolar a partir de las experiencias de los docentes. Educ. Educ. 18 (1), 137-152.

Fernández, I. Álvarez, J. Velásquez, A. \& Ruiz, J. (2002). Actitudes, autoconceptos y expresión emocional en América. Revista Internacional de Ciencias Sociales y Humanidades, SOCIOTAM. 12, 63-74.

Gairín, J. (1990). Las actitudes en educación. Un estudio sobre educación matemática. Barcelona. Editorial Boixareu Universitaria. Recuperado de: https://goo.gl/KpHT32

Huh, J. Delorme, D. \& Reid. L. (2006). Perceived third-person effects and consumer attitudes on preventing and banning DTC advertising. Journal of Consumer Affairs, 40, 90. 
Koehler y Mishra (2009). What is technological pedagogical content knowledge? Contemporary Issues in Technology and Teacher Education, 9 (1), 60-70.

Levent Ç. (2010). Evaluation of the views of preservice teachers taught with Moodle during the course named "instructional technology and material design" on the use of teaching materials. Procedia Social and Behavioral Sciences. 9, 1793-1797.

Levis, D. (2007). Enseñar y aprender con informática/ enseñar y aprender informática. Medios informáticos en la escuela argentina. En Cabello, R. \& Levis D. Edits. (2007). Tecnologías informáticas en la educación a principios del siglo XXI. Buenos Aires: Prometeo. Disponible en https://tecnoeducativas.files.wordpress.com/2010/07/u2-y3-levis_pav06.pdf.

Lucumi, P. \& González, M. (2015). El ambiente digital en la comunicación, la actitud y las estrategias pedagógicas utilizadas por docentes. Revista TED, 37, 109-129.

Lustosa de Oliveira, M. Silva, T. Vega, J. Galembeck, E. (2017). El potencial de las aplicaciones educativas en el proceso de evaluación formativa. Revista Góndola, Enseñanza y Aprendizaje de las Ciencias. 12(2), 99-116.

Martínez G. (2010). Relación entre la actitud, el autoconcepto y los valores con el rendimiento académico de estudiantes universitarios en el marco del espacio europeo de educación superior. Revista académica semestral Cuadernos de Educación y Desarrollo. 2 (19). Recuperado de http://www.eumed.net/ rev/ced/19/jamg.htm

Mosquera, C. (2008). Cambio didáctico en la epistemología y en la práctica docente de profesores universitarios de Química. Memoria para optar al título de Doctor en Ciencias Químicas, Universidad de Valencia, España.

Morales, V. (2006). Medición de actitudes en psicología y en educación. España: Gráficas ORGAM.

Nechita, E. \& Timofti, I. (2011). Increasing Inde- pendence versus Increasing Collaboration with ICT Support. International Conference on Education and Educational Psychology (ICEEPSY 2011). Procedia - Social and Behavioral Sciences. 29, 1508-1517.

Nunnally, J. (1967). Psychometric theory. New York: McGraw-Hill.

Nunnally, J \& Bernstein, I. (1978). Psychometric theory. New York: McGraw-Hill.

Oya, T. \& Sadi, S. (2013). Instructional use of information technologies teachers' Resistance to the use of new Technologies. The International Journal of Technologies in Learning. 19, 61-71.

Páramo, P. \& Otálvaro, G. (2006). Investigación Alternativa: Por una distinción entre posturas epistemológicas y no entre métodos. Cinta moebio, 25, 1 - 7. Recuperado de www.moebio.uchile.cl/25/paramo.htm

Paredes, J. \& Dias, R. (2012). La motivación del uso de las TIC en la formación de profesorado en educación ambiental. Ciência \& Educação, 18(2), 353-368.

Patiño, M. \& Sallé, M. (2013). Las TIC en la enseñanza. Un estudio de casos desde el enfoque biográfico-narrativo. Recuperado de http:// ddd.uab.cat/pub/educar/educar a2014m16v50n1/educar_a2014m1-6v50n1p83.pdf

Schmidt, D. Baran, E. Thompson, A. Mishra, P. Koehler, M. \& Shin T. (2009). Technological Pedagogical Content Knowledge (TPACK): The Development and Validation of an Assessment Instrument for Preservice Teachers. Journal of Research on Technology in Education. 42 (2), 123-149.

Šorgo, A. \& Verčkovnik, T. \& Kocijančič, S. (2010). Information and Communication Technologies (ICT) in Biology Teaching in Slovenian Secondary Schools. Eurasia Journal of Mathematics, Science \& Technology Education, 6(1), 37-46.

Tejedor, F. J. \& García Valcárcel, A. (2006). Competencias de los profesores para el uso de las TIC en la enseñanza. Análisis de sus conocimientos y actitudes. Revista española de 
pedagogía. 233, 21-44.

Tejedor, F. J., García Valcárcel, A., \& Prada, S. (2009). Medida de actitudes del profesorado universitario hacia la integración de las TIC. Comunicar. 33(17), 115-124.

UNESCO, (2013). Enfoques estratégicos de las TICS en educación en América Latina y el Caribe. Santiago de Chile: UNESCO.

Valdés, A. Arreola, C. Angulo, J. Carlos E. \& García, R. (2011). Actitudes de docentes de educación básica hacia las TIC. Revista Internacional de Investigación en Educación, 3(6), $379-392$.
Vera, Tomak. \& Snežana, P. (2014). Biology teachers' attitudes toward computer assisted learning. Archive Biology Scienci. 66(3), 1281-1289.

Yarto, C. (2010). Limitaciones y alcances del enfoque de domesticación de la tecnología en el estudio del teléfono celular. Comunicación y Sociedad. 173-200.

Zapata, M. (2006). La actitud de los docentes ante el diseño instruccional tecnológico. Revista de Educación a Distancia, (15). Recuperado de http://www.redalyc.org/articulo. oa? $\mathrm{id}=54701504$

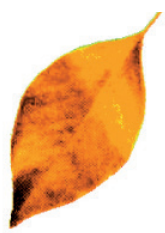

